\title{
Potencialidades e fragilidades da rede de atenção à saiude da mulher no munieipio de Surubim
}

\author{
Potential and weaknesses of the network \\ of women's care in the municipality of Surubim
}

\section{Carla Cristina Franழ̧a de Araújo ${ }^{1}$ Juliana Leão Pontes ${ }^{1}$ Taciana Leão Pontes ${ }^{1}$}

\section{Resumo}

As redes de atenção à saúde da mulher tem como foco impedir mortes maternas e infantis evitáveis além de serem de interesse nacional e internacional no campo da saúde e dos direitos reprodutivos. O objetivo desse ensaio foi realizar uma análise crítica da rede de atenção à saúde da mulher no município de Surubim levando-se em consideração dados relativos à assistência dessa rede disponibilizados pela Secretaria Estadual de Saúde de Pernambuco. Problemas relativos a capacidade de expansão da rede de atenção básica, quantidade de leitos e planejamento familiar foram apontados. Concluiu-se que assim como em Recife e outros municípios, Surubim necessita que os gestores estejam com os olhos voltados para a assistência integral à saúde da mulher.

\section{Abstraet}

Networks of attention to women's health focuses on preventing maternal and child deaths preventable and are of national and international interest in health and reproductive rights. The purpose of this paper was to conduct a critical analysis of the network of health care of women in the municipality of Surubim taking into account data on that network service provided by the State Health Secretariat of Pernambuco. Problems regarding the scalability of the network of primary care, number of beds and family planning were identified. We conclude that as in Recife and other cities, Surubim requires that managers are with their eyes turned to comprehensive health care of women.
Deseritores: SUS, Saúde da Mulher, Sistemas de Saúde.

Keywords: Unified Heath System, Women's Health, Health System

\footnotetext{
'Enfermeiras, Diseentes do Curso de Especializậão em Gestão em Saúde Públiea - Núreleo Integrado de Saúde Coletiva [NISC] - Universidade de Pernambuco [UPE]
}

Para correspondência:

Carla Cristina França de Araújo email:jasielecarla@hotmail.com 


\section{Introdução}

No Brasil, a atenção à mulher na gestação e parto permanece como um desafio para a assistência, no que se refere ao acesso a um atendimento de qualidade e na garantia de integralidade no cuidado ${ }^{1}$.

Apesar da assistência à gestante ser uma das atividades realizadas há mais tempo nos serviços públicos de saúde no país, melhorar a saúde materna e impedir mortes maternas e infantis evitáveis é, ainda, um dos objetivos de maior interesse nacional e internacional no campo da saúde e dos direitos reprodutivos, no qual se discutem quais as medidas necessárias e eficazes para alcançar tal propósito ${ }^{1}$.

Um dos elementos fundamentais para o alcance desses objetivos é a oferta adequada de ações e serviços de saúde. E para que essa oferta adequada se torne realidade, é preciso que exista uma diversidade de serviços de saúde, nos seus diversos níveis de complexidade, articulados entre si, configurando o que denominamos Rede de Atenção à Saúde².

Segundo Mendes, as redes de atenção à saúde são organizações poliárquicas de conjuntos de serviços de saúde, vinculados entre si por uma missão única, por objetivos comuns e por uma ação cooperativa e interdependente, que permitem ofertar uma atenção contínua e integral a determinada população, coordenada pela atenção primária à saúde - prestada no tempo certo, no lugar certo, com o custo certo, com a qualidade certa e de forma humanizada, e com responsabilidades sanitárias e econômicas por esta população ${ }^{2}$.

Entendendo que nem sempre a realidade do município permite que ele possua em seu território todos os serviços de saúde necessários para uma atenção integral à saúde de sua população e respeitando os preceitos da economia de escala, alguns serviços podem e devem ser estruturados para servir de referência regional. Prestando-se também a essa lógica, segundo orientação da Norma Operacional de Assistência à Saúde (NOAS) os municípios foram agrupados em regionais de saúde, considerando algumas características nessa junção.

A idéia de agrupar os municípios em microrregiões de saúde - GERES, surgiu da necessidade de organizar um sistema de saúde que leve em conta a grande heterogeneidade dos municípios e estados brasileiros, tanto do ponto de vista da capacidade instalada em termos de prestação de serviços como na capacidade gerencial existente. A regionalização da assistência à saúde está fundamentada na concepção geral de descentralização com ênfase na regionalização como estratégia de organização hierarquizada dos serviços de saúde, visando a integralidade da atenção e o aumento da equidade na aquisição de recursos e no acesso da população às ações e serviços de saúde, em todos os níveis de atenção com qualidade e resolutividade ${ }^{3}$.

A partir do surgimento da regionalização, se tem a necessidade da criação de redes de atenção à saúde funcionantes e eficazes, porém torna-se necessário um amplo conhecimento sobre o que vem a ser uma rede de atenção, para que não sejam utilizadas de forma errônea.

Desta forma conhecendo a importância do processo de regionalização e da necessidade da formação das redes de atenção à saúde, temos como objetivo neste trabalho analisar a estruturação da rede de atenção à saúde da mulher no município de Surubim.

\section{Marco teórico}

\section{A sittuação de saúde no Brasil}

Para se discutir uma proposta de organização do Sistema Único de Saúde no Brasil, torna-se necessário uma avaliação ou levantamento das necessidades de saúde da população.

$\mathrm{Na}$ perspectiva epidemiológica, o país vivencia uma forma de transição singular, diferente da transição clássica dos países desenvolvidos. Para a análise epidemiológica, vai se considerar o conceito de condições de saúde, agudas e crônicas, que difere da tipologia mais usual que é doenças transmissíveis e doenças e agravos não transmissíveis. Essa tipologia convencional tem sido muito útil nos estudos epidemiológicos, mas observa-se que ela não se presta para referenciar a estruturação dos sistemas de atenção à saúde.

Para organizar os sistemas de atenção à saúde, o mais conveniente é separar as condições agudas, em geral de curso curto e que podem ser respondidas por um sistema reativo e com respostas episódicas, das condições crônicas, que têm curso geralmente longo e que exigem um sistema que responda a elas de forma proativa, contínua e integrada. Por isso, as condições crônicas envolvem todas as doenças crônicas e aquelas doenças transmissíveis de curso longo (tuberculose, hanseníase, HIV/aids e outras), as condições maternas e infantis, os acompanhamentos por ciclos de vida (puericultura, herbicultura e seguimento das 
pessoas idosas), as deficiências físicas e estruturais contínuas (amputações, cegueiras e deficiências motoras persistentes) e os distúrbios mentais de longo prazo ${ }^{4}$.

Uma análise da mortalidade no Brasil indica que, em 1930, as doenças infecciosas respondiam por $46 \%$ das mortes, mas que este valor decresceu para um valor próximo a $5 \%$ em 2000; ao mesmo tempo, as doenças cardiovasculares, que representavam em torno de $12 \%$ das mortes em 1930, responderam, em 2000 , por quase $30 \%$ de todos os óbitos ${ }^{5}$. Em relação à morbidade, medida pela morbidade hospitalar, no ano de 2005, das primeiras quinze causas de internações pelo SUS, nove foram por condições crônicas ${ }^{2}$. A análise da carga de doenças, medida em anos de vida perdidos ajustados por incapacidade, demonstra que $14,7 \%$ dessa carga são por doenças infecciosas, parasitárias e desnutrição; $10,2 \%$, por causas externas; $8,8 \%$, por condições maternas e perinatais e $66,3 \%$, por doenças crônicas ${ }^{6}$.

O somatório das duas últimas, ambas condições crônicas, indica que $75 \%$ da carga de doenças no país são determinados por condições crônicas, o que, ainda, exclui o percentual de doenças transmissíveis de curso longo. A situação epidemiológica brasileira distancia-se da transição epidemiológica clássica ${ }^{7}$ observada nos países desenvolvidos, e tem sido definida, recentemente, como tripla carga de doenças por que envolve, ao mesmo tempo, uma agenda não concluída de infecções, desnutrição e problemas de saúde reprodutiva; o desafio das doenças crônicas e de seus fatores de riscos, como o tabagismo, o sobrepeso, a obesidade, a inatividade física, o estresse e a alimentação inadequada; e o forte crescimento das causas externas ${ }^{8}$.

\section{O processo de regionalizậão}

A regionalização da atenção surgiu após a revolução Russa. Não se pode esquecer que o modelo de regionalização não pode tornar secundário o papel dos municípios. A autoridade sanitária deve ser exercida por todos os entes federados e pela sociedade civil organizada respeitando as competências definidas legalmente para construção de um processo solidário e participativo?.

Todo processo deve ser pautado pela democratização dos cenários e pela busca incessante da prática e do discurso uniforme. Não podemos perder mais tempo, Pernambuco está num vazio histórico o que causou prejuízos sanitários talvez irreparáveis. São quase nove anos de uma regionalização meramente cartorial com um forte poder centralizador do Estado. Este modelo propiciou enormes desvantagem que impedem o SUS de operar com economicidade e qualidade dos serviços que devem ser superadas com urgência. O momento de superar o paradigma da municipalização autárquica e da descentralização estatal chegou?.

Não podemos pensar mais na expansão de serviços com enfoque na oferta de procedimentos. Este modelo fragmentou os serviços de saúde do que decorreram significativas deseconomias de escala e de escopo e como consequência baixa qualidade dos serviços. Neste modelo, o município expande a assistência à saúde sem nenhuma articulação regional, pulverizando recursos públicos e gerando brutais ineficiências. A integralidade da atenção deve ser garantida através de uma rede horizontal coordenada pela atenção primária à saúde. Nos territórios regionais deverão ser ofertados autossuficiência em atenção de média complexidade?.

Este será um espaço privilegiado onde os municípios da microrregião sanitária, em associação entre si e com o apoio da união e da Secretaria Estadual de Saúde, farão a gestão das ações de atenção primária e secundária na lógica de redes de atenção à saúde com base na cooperação gerenciada, gerando, pela sua ação solidária, um excedente cooperativo'.

\section{Redes de atenţão à sauide}

Uma análise dos sistemas de atenção à saúde, feita numa perspectiva internacional, mostra que eles são dominados pelos sistemas fragmentados, voltados para atenção às condições agudas e às agudizações de condições crônicas ${ }^{2}$.

Entende-se por sistema fragmentado aquele onde os serviços de saúde funcionam como "ilhas" de atendimento, sem que exista uma continuidade na assistência e uma responsabilização pelo cuidado do indivíduo. Os sistemas fragmentados têm sido um desastre sanitário e econômico em todo o mundo ${ }^{2}$.

É neste cenário que emerge o conceito de Redes de Atenção Saúde, sendo entendida como uma alternativa que permitem a oferta de uma atenção contínua e integral à determinada população ${ }^{2}$.

A rede constitui-se num conjunto de unidades de diferentes perfis e funções, organizadas de forma articulada e responsáveis pela provisão 
integral de serviços de saúde à população de sua região. Dessa forma, para que efetivamente seja constituída uma rede, duas questões são centrais: a responsabilização pela atenção ao paciente e a articulação efetiva entre as unidades para garantir à população não apenas o acesso nominal, mas a continuidade do cuidado ${ }^{10}$.

\section{Assistêneia integral a saúde da mulher}

As mulheres são a maioria da população brasileira $(50,77 \%)$ e as principais usuárias do Sistema Único de Saúde (SUS). Freqüentam os serviços de saúde para o seu próprio atendimento mas, sobretudo, acompanhando crianças e outros familiares, pessoas idosas, com deficiência, vizinhos, amigos. São também cuidadoras, não só das crianças ou outros membros da família, mas também de pessoas da vizinhança e da comunidade ${ }^{11}$.

A situação de saúde envolve diversos aspectos da vida, como a relação com o meio ambiente, o lazer, a alimentação e as condições de trabalho, moradia e renda. No caso das mulheres, os problemas são agravados pela discriminação nas relações de trabalho e a sobrecarga com as responsabilidades com o trabalho doméstico. Outras variáveis como raça, etnia e situação de pobreza realçam ainda mais essas desigualdades ${ }^{11}$.

As mulheres vivem mais do que os homens, porém adoecem mais frequentemente. A vulnerabilidade feminina frente a certas doenças e causas de morte está mais relacionada com a situação de discriminação na sociedade do que com fatores biológicos ${ }^{11}$.

Os indicadores epidemiológicos do Brasil mostram uma realidade na qual convivem doenças dos países desenvolvidos (cardiovasculares e crônico-degenerativas) com aquelas típicas do mundo subdesenvolvido (mortalidade materna e desnutrição). Os padrões de morbimortalidade encontrados nas mulheres revelam também essa mistura de doenças, que seguem as diferenças de desenvolvimento regional e de classe social ${ }^{11}$.

O Ministério da Saúde, em 2007, considerando que a saúde da mulher é uma prioridade de governo, elaborou o documento "Política Nacional de Atenção Integral à Saúde da Mulher - Princípios e Diretrizes", buscando consolidar os avanços no campo dos direitos sexuais e reprodutivos, com ênfase na melhoria da atenção obstétrica, no planejamento familiar, na atenção ao abortamento inseguro e no combate à violência doméstica e sexual. Agrega, também, a prevenção e o tratamento de mulheres vivendo com $\mathrm{HIV} /$ aids e as portadoras de doenças crônicas não transmissíveis e de câncer ginecológico. Além disso, amplia as ações para grupos historicamente alijados das políticas públicas, nas suas especificidades e necessidades ${ }^{11}$.

A humanização e a qualidade da atenção em saúde são condições essenciais para que as ações de saúde se traduzam na resolução dos problemas identificados, na satisfação das usuárias, no fortalecimento da capacidade das mulheres frente à identificação de suas demandas, no reconhecimento e reivindicação de seus direitos e na promoção do autocuidado $^{11}$.

Os objetivos específicos desta Política são: (a) ampliar e qualificar a atenção clínico-ginecológica, inclusive para as portadoras da infecção pelo HIV e outras DST; (b) estimular a implantação e implementação da assistência em planejamento familiar, para homens e mulheres, adultos e adolescentes, no âmbito da atenção integral à saúde; (c) promover a atenção obstétrica e neonatal, qualificada e humanizada, incluindo a assistência ao abortamento em condições inseguras, para mulheres e adolescentes; (d) promover a atenção às mulheres e adolescentes em situação de violência doméstica e sexual; (e) promover, conjuntamente com o PNDST/AIDS, a prevenção e o controle das doenças sexualmente transmissíveis e da infecção pelo $\mathrm{HIV} /$ aids na população feminina; (f) reduzir a morbimortalidade por câncer na população feminina; (g) implantar um modelo de atenção à saúde mental das mulheres sob o enfoque de gênero; (h) implantar e implementar a atenção à saúde da mulher no climatério; (i) promover a atenção à saúde da mulher na terceira idade; (j) promover a atenção à saúde da mulher negra; (k) promover a atenção à saúde das trabalhadoras do campo e da cidade; (l) promover a atenção à saúde da mulher indígena; (m) promover a atenção à saúde das mulheres em situação de prisão, incluindo a promoção das ações de prevenção e controle de doenças sexualmente transmissíveis e da infecção pelo $\mathrm{HIV} /$ aids nessa população e (n) fortalecer a participação e o controle social na definição e implementação das políticas de atenção integral à saúde das mulheres.

\section{Deserição e análise referente a saúde da mulher em Surubim}

O município de Surubim localiza-se a uma latitude $07^{\circ} 49^{\prime} 59^{\prime \prime}$ sul e a uma longitude $35^{\circ} 45^{\prime} 17^{\prime \prime}$ oeste, estando a uma altitude de 220 metros acima do nível do mar. Sua população estimada em 2004 era de 52329 habitantes. Possui uma área de 254,94 
$\mathrm{km}^{2}$. Limita-se com os municípios de Casinhas e Vertente do Lério (norte), Frei Miguelinho e Santa Maria do Cambucá (oeste), Salgadinho, João Alfredo e Bom Jardim (leste) e Riacho das Almas e Cumaru (sul).

Com relação à saúde o município é classificado como município sede módulo assistencial, referência para média complexidade desde 2006 e apresentou adesão ao pacto pela saúde em 2009. Possui: 226 leitos hospitalares, todos disponíveis para pacientes do Sistema Único de Saúde, mortalidade infantil de 82,9 por mil e esperança de vida ao nascer de 63,6 anos.

Inicialmente, podemos observar que $\mathrm{O}$ estimado para as ESF, seria de 23 e temos no município apenas 20, ou seja, ainda existe uma capacidade de expansão de 03 unidades, citei as unidades básicas, pois é nela que se realizam várias ações referentes à saúde da mulher, tais como: planejamento familiar, pré-natal e consulta puerperal.

\begin{tabular}{|l|c|}
\hline \multicolumn{1}{|c|}{ Unidades e/ou Leitos } & Números Existentes \\
\hline $\begin{array}{l}\text { Equipes de saúde } \\
\text { da família }\end{array}$ & 20 \\
\hline $\begin{array}{l}\text { Leitos de } \\
\text { Pré-parto }\end{array}$ & 05 \\
\hline $\begin{array}{l}\text { Leitos } \\
\text { Ginecológicos }\end{array}$ & 04 \\
\hline $\begin{array}{l}\text { Leitos Obstétricos } \\
\text { Clínicos }\end{array}$ & 34 \\
\hline $\begin{array}{l}\text { Leitos Obstétricos } \\
\text { Cirúrgicos }\end{array}$ & 04 \\
\hline $\begin{array}{l}\text { Serviços que } \\
\text { realizam Laqueadura }\end{array}$ & 00 \\
\hline $\begin{array}{l}\text { USG de } 1^{\circ} \text { e } 2^{\circ} \text { nível } \\
\text { 02(SUS) }\end{array}$ \\
\hline $\begin{array}{l}\text { Leitos Obstétricos } \\
\text { por Hospitais }\end{array}$ & $\begin{array}{c}\text { Hospitas) São Luiz: 32 } \\
\text { Hospital Municipal de Surubim: 06 } \\
\text { Maternidade Estefânia Farias: 00 }\end{array}$ \\
\hline
\end{tabular}

Quadro 1. Número de leitos relativos a rede de atenção à saúde da mulher em Surubim - Pernambuco, Brasil/2008.

Não se sabe se essas ações são realmente desenvolvidas no município e se são realizadas com qualidade, mas um número no quadro 1 já nos aponta algo que precisa melhorar ou existir, que é a inexistência de leitos ou serviços que realizem a laqueadura tubária, pois como a portaria de Assistência ao Planejamento Familiar diz que deve ser garantida a mulher ou família todos os métodos contraceptivos determinados pelo Ministério da Saúde, então uma mulher, por exemplo, maior que
25 anos, com 05 filhos todos vivos, deveria ser uma forte candidata a uma laqueadura.

Outro ponto que me chamou bastante atenção é o fato do município possuir uma maternidade, ou um prédio que seria destinado à maternidade, mas que não funciona e na verdade os leitos obstétricos e ginecológicos estão englobados nos dois hospitais gerais do município. Deve ser garantido a mulher um ambiente apenas obstétrico, com profissionais apenas voltados para a obstetrícia, ou seja, profissionais capacitados ou até mesmo especializados, com a garantia de um parto humanizado, onde a mulher é a protagonista do parto. É fato que em um hospital geral isso se torna impossível, pois na maioria das vezes a enfermeira ou enfermeiras existentes são responsáveis por todo hospital atuando apenas como uma supervisora ao invés de um profissional assistencial.

Assim como em outros municípios, em Surubim os recursos destinados à saúde são limitados e insuficientes para a oferta de um atendimento integral e de qualidade ao usuário, assim, sua alocação deve considerar a priorização em ações com maior poder de resolução.

Além disso, devemos continuar denunciando o subfinanciamento que existe na saúde, para que a situação caótica que temos em muitas unidades hoje não seja atribuída a uma ineficiência da gestão pública, discurso muito utilizado por aqueles que pretendem privatizar a gestão da saúde. Precisamos também estimular uma maior articulação interfederativa. As decisões precisam ser descentralizadas e a qualificação da gestão com instituição da carreira SUS necessita ser priorizada.

\section{Conclusões}

Portanto, conclui-se que assim como em Recife e outros municípios, Surubim necessita que os gestores estejam com os olhos voltados para a assistência integral à saúde da mulher e que seja garantida essa assistência de qualidade e com profissionais especializados, para que possamos reverter o quadro epidemiológico do nosso país em relação as mulheres e que a autonomia desta mulher enquanto colaboradora do processo de saúde doença seja resgatado. 


\section{Referêneias}

1. Jacob SS, Guilherme CJ, Lago Giacomo, T. O Programa de Humanização no Pré-natal e Nascimento do Ministério da Saúde no Brasil: resultados iniciais. Cad. Saúde Pública [serial on the Internet]. 2004 Oct [cited 2011 Aug 21] ; 20(5): 1281-1289.

2. Mendes EV. As redes de atenção à saúde. Belo Horizonte: Escola de Saúde Pública de Minas Gerais; 2009.

3. Secretaria Estadual de Saúde de Pernambuco. Plano Diretor de Regionalização, 2006.

4. Organização Mundial da Saúde. Cuidados inovadores para condições crônicas: componentes estruturais de ação. Brasília: Organização Mundial da Saúde; 2003.

5. Silva JB, Gomes FBC, Cezário AC, Moura L. Doenças e agravos não transmissíveis: bases epidemiológicas. In: Rouquayrol MZ, Almeida Filho N. Epidemiologia e saúde. $6^{\text {a }}$ ed. Rio de Janeiro: Medsi; 2006.

6. Shramm JMA, Oliveira AF, Leite IC, Valente JG, Gadelha AMJ, Portela MC, Campos MR. Transição epidemiológica e o estudo de carga de doença no Brasil. Cien Saude Colet 2004; 9:987-908.

7. Omran AR. The epidemiologic transition: a theory of the epidemiology of population change. Milbank Mem. Fund 1971; 49:509-583.

8. Frenk J. Bridging the divide: comprehensive reform to improve health in Mexico. Nairobi: Commission on Social Determinants of Health; 2006.

9. Secretaria do Estadual de Saúde de Pernambuco - SES/ PE. Análise de Situação de Saúde da Região de Limoeiro em Pernambuco. 193p. 2009.

10. Kuschnir RC, Chorny AH, Lira AML. Gestão dos sistemas e serviços de saúde - Florianópolis: Departamento de Ciências da Administração / UFSC; [Brasília]: CAPES : UAB, 2010.

11. Brasil. Ministério da Saúde. Secretaria de Atenção à Saúde. Departamento de Ações Programáticas Estratégicas. Política nacional de atenção integral à saúde da mulher: princípios e diretrizes / Ministério da Saúde, Secretaria de Atenção à Saúde, Departamento de Ações Programáticas Estratégicas. - Brasília: Editora do Ministério da Saúde, 2007.82 p. 